\title{
Prospects for Backtracing 1I/`Oumuamua and Future Interstellar Objects
}

Qicheng Zhang (i)

Division of Geological and Planetary Sciences, California Institute of Technology, Pasadena, CA 91125, USA; qicheng@cometary.org Received 2017 November 30; revised 2017 December 18; accepted 2017 December 19; published 2018 January 3

\begin{abstract}
1I/'Oumuamua is the first of likely many small bodies of extrasolar origin to be found in the solar system. These interstellar objects (ISOs) are hypothesized to have formed in extrasolar planetary systems prior to being ejected into interstellar space and subsequently arriving at the solar system. This paper discusses necessary considerations for tracing ISOs back to their parent stars via trajectory analysis and places approximate limits on doing so. Results indicate that the capability to backtrace ISOs beyond the immediate solar neighborhood is presently constrained by the quality of stellar astrometry, a factor poised for significant improvement with upcoming Gaia data releases. Nonetheless, prospects for linking 1I or any other ISO to their respective parent stars appear unfavorable on an individual basis due to gravitational scattering from random stellar encounters, which limit traceability to the past few tens of millions of years. These results, however, do not preclude the possibility of occasional success, particularly after considering the potential for observational bias favoring the discovery of younger ISOs, together with the anticipated rise in the ISO discovery rate under forthcoming surveys.
\end{abstract}

Key words: astrometry - local interstellar matter - minor planets, asteroids: individual (1I/'Oumuamua) solar neighborhood - stars: kinematics and dynamics

\section{Introduction}

1I/'Oumuamua (henceforth, 1I) is the first small body of definitive extrasolar origin to be identified within the solar system. 1I was originally discovered by the Panoramic Survey Telescope And Rapid Response System (Pan-STARRS; Kaiser et al. 2002), and announced as C/2017 U1 (PANSTARRS) on 2017 October 25 as a comet on an improbable trajectory for a solar system object, with eccentricity $e \approx 1.2$ and hyperbolic excess speed $v_{\infty} \approx 26 \mathrm{~km} \mathrm{~s}^{-1}$ (Williams 2017b). The object was subsequently redesignated A/2017 U1 after follow-up observations revealed a stellar morphology (Williams 2017a), before it was finally given its present designation on 2017 November 6 under a new dedicated naming scheme for interstellar objects (ISOs; Williams 2017c).

The anticipated arrival of the Large Synoptic Survey Telescope (LSST; Tyson 2002), to be capable of quickly finding fainter objects over wider fields than current surveys, is poised to dramatically expand the catalog of similar ISOs in the solar system (Trilling et al. 2017). ISOs, whose hypothesized existence far predates the discovery of 1I (Sekanina 1976; Sen \& Rana 1993; Engelhardt et al. 2017), are believed to have formed in extrasolar planetary systems before being ejected into interstellar space. Spectral and photometric observations of 1I show an absence of ultra-red material typical of outer solar system objects, suggesting it either formed or subsequently evolved within the snow line of its parent star (Jewitt et al. 2017; Meech et al. 2017; Ye et al. 2017). In the absence of a larger sample, the characteristics established for 1I may be assumed to be representative of typical ISOs.

Given the significant levels of ongoing star formation activity in the Milky Way (Kennicutt \& Evans 2012) through which the ISO population is presumably supplied, it seems conceivable that at least a fraction of discovered ISOs could potentially be linked back to their parent stars. Attempts to link 1I to stars in the immediate solar neighborhood have yielded no obvious candidates (Mamajek 2017; Ye et al. 2017). Additional searches by Gaidos et al. (2017), Portegies Zwart et al. (2017), Dybczyński \& Królikowska (2017), and Feng \& Jones (2017) considered a wider selection of stars, particularly from the Tycho-Gaia Astrometric Solution (TGAS; Michalik et al. 2015), but mostly focused on the subset of stars with available radial velocity data for which encounter time and velocity can be constrained. No definitive point of origin for 1I was identified by any of these searches, though numerous plausible options have been proposed.

The following sections discuss considerations for backtracing ISOs, including 1I, to their parent stars in the context of astrometric uncertainties associated with both the ISO and of the candidate stars. Approximate bounds are placed on the potential for success in search attempts for a given ISO, including 1I, in both the present and future. Finally, a past encounter search method that does not require radial velocity measurements is introduced and tested for $1 \mathrm{I}$.

\section{Scattering Timescale}

The capacity to predict or, in this instance, backtrace an ISO in a chaotic dynamical system like the Milky Way galaxy is fundamentally limited by incomplete information on the state of the system. Specifically, the close approach of an ISO to a star with gravitational parameter $\mu_{*} \equiv G M_{*}$ at relative speed $v_{\infty}$ will scatter the ISO by an angle $\Delta \theta$ given by Equation (1) that is sensitive to the impact parameter $b$ of the encounter.

$$
\sin \frac{\Delta \theta}{2}=\left(1+\left(\frac{b v_{\infty}^{2}}{\mu_{*}}\right)^{2}\right)^{-1 / 2} .
$$

Over a typical interstellar distance $L$ between encounters, even a small $\Delta \theta \ll 1$ can produce a large perpendicular displacement $\Delta y \approx L \Delta \theta \gtrsim b$, resulting in successive stellar encounters becoming completely unpredictable. In the $\Delta \theta \ll 1 \Rightarrow b v_{\infty}^{2} \gg \mu_{*}$ limit, Equation (1) simplifies to

$$
\Delta \theta=\frac{2 \mu_{*}}{b v_{\infty}^{2}}
$$


Consider the maximum $b=b_{0}$ of a stellar encounter for which the following encounter of similar $b_{0}$ becomes unpredictable, with $\Delta y \sim b_{0}$. The average distance between two encounters of impact parameter below $b_{0}$ in a region of uniform stellar density $n_{*}$ is given by the corresponding meanfree path $L_{0} \sim\left(n_{*} \pi b_{0}^{2}\right)^{-1}$. In addition, encounter speed $v_{\infty}$ generally satisfies $v_{\infty} \sim u_{*}$, the stellar velocity dispersion, for a recently introduced ISO. This approximation is valid as long as the ISO is a galactic disk object, as 1I presently is. Then,

$$
\Delta y=\frac{2 \mu_{*}}{\pi b_{0}^{3} n_{*} u_{*}^{2}}
$$

and $\Delta y \sim b_{0}$ when

$$
b_{0} \sim\left(\frac{2 \mu_{*}}{\pi n_{*} u_{*}^{2}}\right)^{1 / 4}
$$

which corresponds to a mean-free path

$$
L_{0} \sim \frac{u_{*}}{\left(2 \pi n_{*} \mu_{*}\right)^{1 / 2}},
$$

which is crossed over a scattering timescale

$$
\tau_{0} \sim \frac{L_{0}}{u_{*}} \sim\left(2 \pi n_{*} \mu_{*}\right)^{-1 / 2}
$$

This scattering timescale $\tau_{0}$ serves as an approximate upper bound on the duration over which an ISO can reliably be traced given near-perfect astrometry of both the ISO and of stars in the galaxy. Note that to a factor of the order of unity, the $\tau_{0}$ given by Equation (6) also serves as a scattering timescale over which stars, which are similarly scattered in encounters with other stars, can reliably be traced.

The solar neighborhood features a wide distribution of $\mu_{*}$ with relative abundance per mass interval $\xi\left(\mu_{*}\right)$. A characteristic $\mu_{*}$ occurs at the peak of the $\xi\left(\mu_{*}\right) \mu_{*}$ distribution, with stars near this $\mu_{*}$ most strongly constraining $L_{0}$ and $\tau_{0}$ as both values are minimized at maximum $n_{*} \mu_{*}$. Chabrier (2003) finds characteristic values near $\mu_{*} \sim 0.2 G M_{\odot}$ for stars and close multi-star systems (which are effectively equivalent to single stars in scattering ISOs) in the Milky Way galaxy.

TGAS, which is nearly complete for stars of this $\mu_{*}$ within a few parsecs of the Sun, has a stellar density $n_{*} \sim 0.15 \mathrm{pc}^{-3}$ in this region (Michalik et al. 2015). In addition, the local stellar velocity dispersion is estimated to be $u_{*} \sim 20 \mathrm{~km} \mathrm{~s}^{-1}$ (Huang et al. 2015). A trajectory through stellar environments comparable to the solar neighborhood corresponds to a scattering timescale of $\tau_{0} \sim 30 \mathrm{Myr}$, with $L_{0} \sim 700 \mathrm{pc}$ and $b_{0} \sim 0.05 \mathrm{pc}$. This result is consistent with that of numerical simulations by Dybczyński \& Królikowska (2017), who find gravitational perturbations from nearby stars to be insignificant.

Suppose ISOs have been produced at a constant rate over the past $t_{\max } \sim 10 \mathrm{Gyr}$ in the Milky Way galaxy, and are spatially distributed independently of age. Under this simple model, a fraction $\tau_{0} / t_{\max } \sim 3 \times 10^{-3}$ of these ISOs are expected to be realistically traceable to their parent stars given near-optimal astrometry together with an accurate model of the galactic potential.

Note, however, that observational biases favoring young ISOs could significantly amplify the fraction of traceable ISOs by reducing the effective $t_{\max }$. Consider an extreme example where a hypothetical mechanism that removes each ISO from the galaxy after it spends $t_{\max }=10 \mathrm{Myr}$ in interstellar space.
Since $t_{\max }<\tau_{0}$ for this example, all discovered ISOs are younger than the scattering timescale, so have likely never been scattered and remain traceable with accurate astrometry.

Feng \& Jones (2017) propose a more realistic mechanism by which older ISOs are scattered out of the galactic disk entirely after repeated stellar encounters, thus suppressing their discovery rate. Given that this mechanism requires repeated scattering events, it must operate over a timescale $t_{\max } \gg \tau_{0}$, and so, still only a minute fraction of discovered ISOs are likely to be traceable in the absence of stronger bias.

\section{Astrometric Considerations}

In practice, astrometric uncertainties may limit the range over which a given ISO can be linked to its parent star well short of the scattering timescale derived above. Two distinct classes of uncertainties affect the ability to trace an ISO's trajectory, and both must be considered when attempting to do so:

1. Uncertainties in the ISO's trajectory.

2. Uncertainties in stellar trajectories.

The first is initially large at the ISO's discovery when few observations are available, then decreases over the course of its passage through the solar system as additional observations refine its trajectory, and finally settles at a minimum value once the ISO becomes unobservable. The second decreases more slowly as improved stellar astrometric catalogs become available, but may continue to decrease into the foreseeable future with data from missions like Gaia. The consequences of each class on the potential for backtracing an ISO are discussed separately below.

\subsection{ISO Trajectory Uncertainties}

Consider, for now, only the uncertainty in the trajectory of the ISO. In practice, the relevant uncertainty is largely in approach velocity, with the uncertainty distribution typically taking the form of a flattened ellipsoid aligned edge-on with the nominal velocity. Let $\Delta \phi_{\text {iso }}$ be the characteristic fractional uncertainty in velocity. If $\Delta \phi_{1}, \Delta \phi_{2}$, and $\Delta \phi_{3}$ are the semi-axes of the uncertainty ellipsoid, then $\Delta \phi_{\text {iso }}$ is well-represented by Equation (7) - chosen such that $\pi \Delta \phi_{\text {iso }}^{2}$ represents a typical cross sectional area for the ellipsoid as approximated by Thomsen's formula, which is generally most accurate with $p \approx 1.6075$. $^{1}$

$$
\Delta \phi_{\text {iso }} \sim\left(\frac{\Delta \phi_{1}^{p} \Delta \phi_{2}^{p}+\Delta \phi_{2}^{p} \Delta \phi_{3}^{p}+\Delta \phi_{1}^{p} \Delta \phi_{3}^{p}}{3}\right)^{1 / 2 p} .
$$

The corresponding uncertainty in the ISO's position $\Delta l$ is related to its distance $r$ from the Sun by $\Delta l \sim r \Delta \phi_{\text {iso }}$. Given a uniform stellar density $n_{*}$, the mean-free path for a random overlap in position between the ISO and a star is $L \sim\left(\pi \Delta l^{2} n_{*}\right)^{-1} \sim\left(\pi r^{2} \Delta \phi_{\text {iso }}^{2} n_{*}\right)^{-1}$. The nearest random overlap is expected to occur when $r \sim L \rightarrow L_{1}$ for which

$$
L_{1} \sim\left(\pi \Delta \phi_{\mathrm{iso}}^{2} n_{*}\right)^{-1 / 3},
$$

\footnotetext{
http://www.numericana.com/answer/ellipsoid.htm
} 


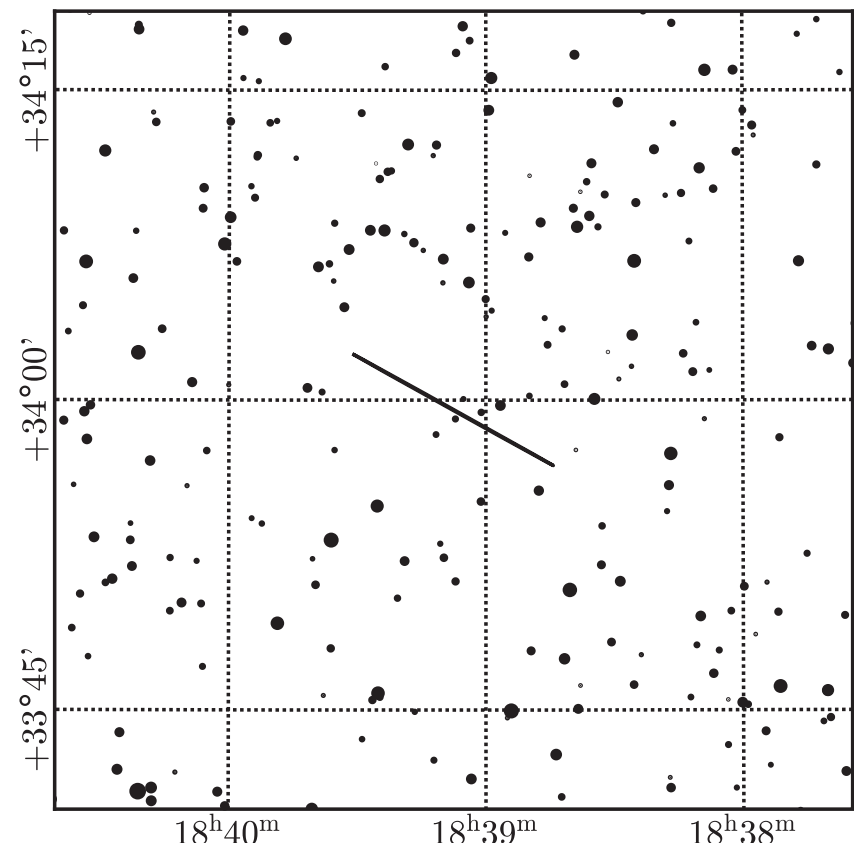

Figure 1. Highly elongated $3 \sigma$ uncertainty ellipse (diagonal bar) for the origin direction of 1I from JPL Small Body Database on 2017 November 20 overlaid on a map of Gaia DR1 stars (epoch 2015.0) with $G$ magnitude $<14$ (Gaia Collaboration et al. 2016b).

corresponding to a limiting timescale of

$$
\tau_{1} \sim \frac{L_{1}}{u_{*}} \sim\left(\pi \Delta \phi_{\mathrm{iso}}^{2} n_{*} u_{*}^{3}\right)^{-1 / 3} .
$$

Beyond $L_{1}$ and $\tau_{1}$, stars passing nowhere near the ISO are expected to begin crossing the position uncertainty ellipsoid by chance alone.

As of 2017 November 20, the JPL Small Body Database orbital solution for $1 \mathrm{I}^{2}$ indicates a $3 \sigma$ uncertainty ellipse for the origin direction of $1 \mathrm{I}$ with semi-axes $\Delta \phi_{1} \times \Delta \phi_{2}=331^{\prime \prime} \times 1^{\prime \prime} 5$ centered on the J2000.0 coordinates $18^{\mathrm{h}} 39^{\mathrm{m}} 7^{\mathrm{s}} .5,33^{\circ} 59^{\prime \prime} 3^{\prime \prime} 2$. Figure 1 plots this flattened uncertainty ellipse - a cross section of the full uncertainty ellipsoid-over a map of the present-day positions of bright stars from Gaia DR1 for context. The corresponding uncertainty in radial velocity $v_{\infty}$ is $0.225 \%$, which subtends an angle $\Delta \phi_{3}=464^{\prime \prime}$. Equation (7) gives $\Delta \phi_{\text {iso }} \sim$ $280^{\prime \prime}$, which places limits of $L_{1} \sim 100 \mathrm{pc}$ and $\tau_{1} \sim 5 \mathrm{Myr}$ for $1 \mathrm{I}$.

Note that for $\Delta \phi_{\text {iso }} \ll \Delta \phi_{\text {sc }} \sim 15^{\prime \prime}, L_{1} \gg L_{0}$ and $\tau_{1} \gg \tau_{0}$. In this scattering-limited regime, further improvements to the ISO trajectory will not appreciably extend the range and period over which it can be accurately be tracked, which are ultimately limited by its most recent scattering encounter. Backtracing is therefore only possible for an ISO whose last scattering event corresponds to its departure from its origin system.

An ISO ejected from within or near the snow line, as 1I may have been (Jewitt et al. 2017; Meech et al. 2017; Ye et al. 2017), would appear to originate in an encounter with its parent stars at a periastron distance $q \lesssim \delta_{\text {snow }}$, where $\delta_{\text {snow }}$ is the snow line distance. Consider $\delta_{\text {snow }} \lesssim 10$ au, which holds through the planetesimal formation process for stars of $\mu_{*} \lesssim 3 G M_{\odot}$ (Kennedy \& Kenyon 2008). Then, for any ISO originating from $r \gg 1$ pc, $q$ subtends an angle of $\phi_{q} \ll 10^{\prime \prime}<\Delta \phi_{\text {sc }}$. At $\Delta \phi_{\text {iso }} \sim \Delta \phi_{\text {sc }}$, the ISO's departure from its origin system

\footnotetext{
https://ssd.jpl.nasa.gov/sbdb.cgi?orb $=1 ;$ sstr $=1 \mathrm{I}$
}

would be indistinguishable from an exact encounter with the parent star, where $q=0$.

The identification and parameters of the last scattering event at time $t_{\mathrm{ls}}$ before present become well-constrained once $\tau_{1} \gg t_{\mathrm{ls}}$. Since $t_{\mathrm{ls}} \lesssim \tau_{0}$ if the apparent last scattering is the ISO origin and $t_{\mathrm{ls}} \sim \tau_{0}$ if not, ISO astrometry should aim to reduce uncertainties to $\Delta \phi_{\text {iso }} \ll \Delta \phi_{\text {sc }} \sim 15^{\prime \prime}$ for $\tau_{1} \gg \tau_{0} \sim 30 \mathrm{Myr}$. Astrometry of such precision is sufficient to establish the encounter associated with the ISO's departure as improbable for a random encounter, should this event be its most recent scattering encounter.

\subsection{Stellar Motion Uncertainties}

The $L_{1}$ and $\tau_{1}$ limits serve as bounds to the long-term prospects of backtracing a particular ISO after astrometric observations of the ISO have concluded. In theory, the quality of the stellar astrometry being searched can continue to improve long after the ISO has left the solar system, and so uncertainties in this data may become negligible in the distant future.

However, an actual backtrace done in the present day is greatly affected by the quality and completeness of the existing stellar astrometry data. Uncertainties in this data increase the effective cross section for a possible encounter. Presently, the largest source of stellar astrometry is the Gaia mission (Gaia Collaboration et al. 2016a). Its first data release, Gaia DR1 (Gaia Collaboration et al. 2016b) contains 1,142,679,769 stars, of which, only the 2,057,050 stars comprising the TGAS subset (Michalik et al. 2015) include a full five-parameter astrometric solution.

The typical $3 \sigma$ proper motion uncertainty among all TGAS stars is $\epsilon_{\mathrm{pm}} \sim \Delta \phi_{\mathrm{pm}} u_{*} / r \sim 4$ mas $\mathrm{yr}^{-1}$, and the corresponding parallax uncertainty $\epsilon_{\mathrm{plx}} \sim 1$ mas. Let $\alpha \equiv 1^{\prime \prime} \mathrm{pc}$ be the proportionality constant relating parallax and $r_{0}^{-1}$. Then, uncertainty in $r_{0}$ is $\Delta r_{0} \sim r_{0}^{2} \epsilon_{\mathrm{plx}} / \alpha \sim r^{2} \epsilon_{\mathrm{plx}} / \alpha$, which corresponds to a relative uncertainty $\Delta \phi_{r 0} \sim r_{0} / r \sim r \epsilon_{\mathrm{plx}} / \alpha$.

Additionally, radial velocity $u_{r}$-not provided by TGAS - is also necessary to determine the timing and relative speed of a close encounter between an ISO and a star. Let uncertainty in $u_{r}$ as a fraction of speed be $\Delta \phi_{\mathrm{rv}}$, so total uncertainty in the radial direction becomes $\Delta \phi_{r} \sim\left(\Delta \phi_{r 0}^{2}+\Delta \phi_{\mathrm{rv}}^{2}\right)^{1 / 2}$. Then, $\Delta \phi_{r}$ and $\Delta \phi_{\mathrm{pm}}$ are combined in an analogous fashion to Equation (7), for an ellipsoid of $\Delta \phi_{\mathrm{pm}} \times \Delta \phi_{\mathrm{pm}} \times \Delta \phi_{r}$, into a characteristic stellar uncertainty

$$
\Delta \phi_{*} \sim\left(\frac{\Delta \phi_{\mathrm{pm}}^{2 p}+2 \Delta \phi_{\mathrm{pm}}^{p} \Delta \phi_{r}^{p}}{3}\right)^{1 / 2 p} .
$$

Combining $\Delta \phi_{*}$ with $\Delta \phi_{\text {iso }}$ gives an effective total relative uncertainty $\Delta \phi \sim\left(\Delta \phi_{\text {iso }}^{2}+\Delta \phi_{*}^{2}\right)^{1 / 2}$. Then, Equation (8) generalizes to

$$
L_{2} \sim\left(\pi \Delta \phi^{2} n_{*}\right)^{-1 / 3}
$$

Consider the limit imposed by uncertainties in the five standard astrometric parameters (i.e., excluding radial velocity), with $\Delta \phi \sim \Delta \phi_{*}$ and $\Delta \phi_{r} \sim \Delta \phi_{r 0}$ at $r \sim L_{2} \rightarrow L_{2}^{\prime}$. Define $\hat{\epsilon}_{\mathrm{pm}} \equiv \epsilon_{\mathrm{pm}} \alpha / u_{*}$, the proper motion uncertainty in a form 


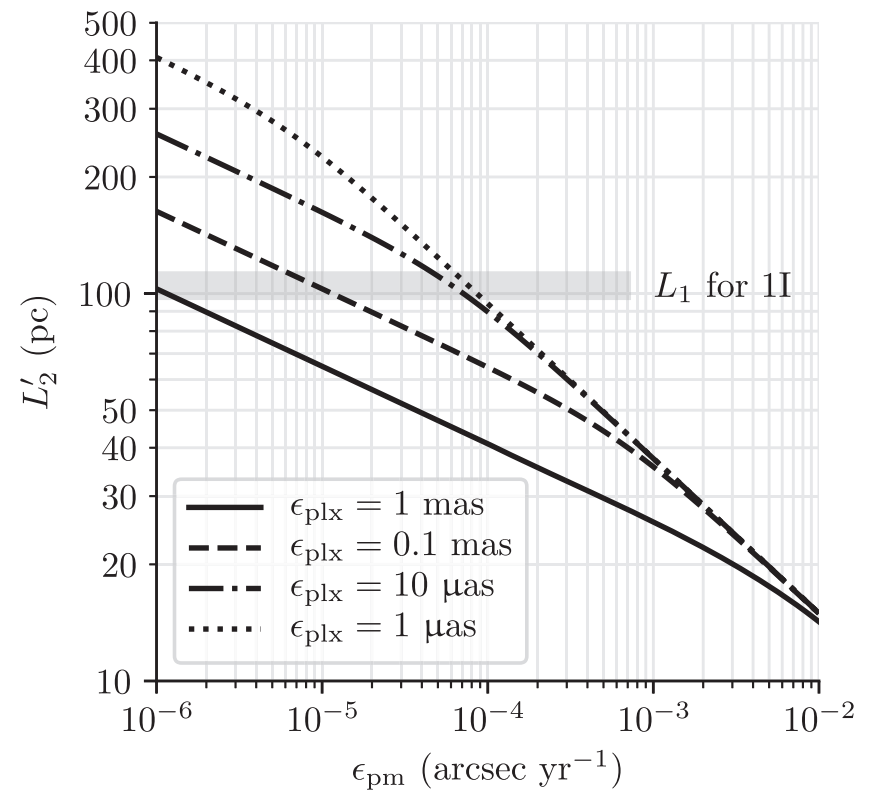

Figure 2. Limiting distance $L_{2}^{\prime}$ for tracing ISOs, while constrained by $3 \sigma$ uncertainties in stellar proper motion $\epsilon_{\mathrm{pm}}$ and parallax $\epsilon_{\mathrm{plx}}$, estimated by Equation (12). The horizontal bar marks the corresponding $L_{1}$ limit from uncertainties in 1I's trajectory, as of 2017 November 20, which become constraining for points above this bar.

that can be related to $\epsilon_{\mathrm{plx}}$. For TGAS, $\hat{\epsilon}_{\mathrm{pm}} \sim 1$ mas $\sim \epsilon_{\mathrm{plx}}$. Then,

$$
L_{2}^{\prime} \sim\left(\frac{\alpha^{2}}{\pi n_{*}}\left(\frac{3}{\hat{\epsilon}_{\mathrm{pm}}^{2 p}+2 \hat{\epsilon}_{\mathrm{pm}}^{p} \epsilon_{r 0}^{p}}\right)^{1 / p}\right)^{1 / 5} .
$$

Figure 2 illustrates the variation in $L_{2}^{\prime}$ with $\epsilon_{\mathrm{pm}}$ and $\epsilon_{\mathrm{plx}}$, and shows the potential growth of the traceable region with improved stellar astrometry in the future. For typical TGAS stars, $L_{2}^{\prime} \sim 20 \mathrm{pc}$, corresponding to $\tau_{2}^{\prime} \sim L_{2} / u_{*} \sim 1 \mathrm{Myr}$. However, stars of $r_{0}<L_{2}^{\prime}$ are predominantly part of the Hipparcos subset of TGAS, which has a characteristic $\epsilon_{\mathrm{pm}} \sim 1 \mathrm{mas} \mathrm{yr}^{-1}$. Using this improved $\epsilon_{\mathrm{pm}}$ raises the limits to $L_{2}^{\prime} \sim 30 \mathrm{pc}$ and $\tau_{2}^{\prime} \sim 1.5 \mathrm{Myr}$.

ISOs can therefore be reliably traced with TGAS only to encounters with nearby stars of $r \ll L_{2}^{\prime} \sim 30 \mathrm{pc}$. Random stars with no physical association to the ISO of interest are expected to be matched beyond this region-the immediate solar neighborhood - and cannot be reliably distinguished from a star actually encountered by the ISO, let alone its true origin.

\section{Linear Backtracing}

Radial velocity often contributes significantly to the uncertainty in the state vector of a star, which is necessary to run a general backtracing simulation involving nonlinear perturbations. Moreover, these measurements are available for only a small subset of stars in TGAS. However, while radial velocity is imperative to constrain the circumstances of an encounter, its value often has minimal effect on whether an encounter occurs at all. Five-parameter stellar astrometric solutions are sufficient to identify encounters with the ISO, provided data uncertainties permit the approximation of the motion of the ISO and all considered stars as linear.

Linear motion is a valid approximation over a timescale $\tau_{\text {lin }}$ such that its fraction of the galactic dynamical time, estimated as the solar orbital period $\tau_{\text {dyn }} \sim 200 \mathrm{Myr}$ (Innanen et al. 1978), is comparable to $\Delta \phi$ :

$$
\frac{\tau_{\text {lin }}}{\tau_{\text {dyn }}} \sim \Delta \phi .
$$

Consider constraints placed by the astrometric uncertainties $\Delta \phi_{\mathrm{pm}}$ and $\Delta \phi_{\mathrm{plx}}$. The condition $r \ll L_{\mathrm{lin}} \sim u_{*} \tau_{\mathrm{lin}}$ is then satisfied as long as $\left(\hat{\epsilon}_{\mathrm{pm}}^{2}+\epsilon_{\mathrm{plx}}^{2}\right)^{1 / 2} \gg \alpha\left(u_{*} \tau_{\mathrm{dyn}}\right)^{-1} \sim 0.2 \mathrm{mas}$, a condition that always holds for TGAS. Approximating motion as linear therefore should not appreciably increase the total uncertainty, given present stellar proper motion uncertainties. Note, however, that the preceding statement may cease to hold for future Gaia data releases should proper motion uncertainties be improved by an order of magnitude or more as anticipated.

Under the linear motion approximation, two geometric criteria are needed to isolate the stars with which the ISO may potentially have had an exact encounter $(b=0)$.

1. The apparent trajectory of every star lies on a great circle. Proper motion uncertainties expand the single great circle into a distribution of possible great circle trajectories spanning a pair of wedges, one leading and one trailing the star's motion. Only those stars where the trailing wedge sufficiently overlaps the ISO origin uncertainty ellipse (as shown for 1I in Figure 1) can potentially have had an encounter with the ISO.

2. Let $\tilde{u}$ be the magnitude of a star's proper motion, and $\theta_{0 *}$ be the angular separation between the star's current position, and the point where it crosses the ISO uncertainty ellipse. Only stars with $r \tilde{u} \equiv u_{\perp}=v_{\infty} \sin \theta_{0 *}$, within data uncertainties, crossed the ISO uncertainty ellipse at the moment the ISO was at the same distance as the star, and thus correspond to an encounter in physical space.

The time and distance scales for a random star to satisfy the first criterion is set by the mean-free path to an encounter on the surface of the celestial sphere. Within a distance $r$, there are $N$ stars:

$$
N \sim \frac{4}{3} \pi r^{3} n_{*}
$$

The angular number density on the celestial sphere is $n_{\Omega} \sim(4 \pi)^{-1} N$, and the corresponding encounter cross section is $\sigma_{\Omega} \sim 2 \Delta \phi_{\mathrm{pm}}$. The resulting mean-free path is

$$
\bar{\theta} \sim\left(n_{\Omega} \sigma_{\Omega}\right)^{-1} \sim \frac{3}{2 r^{3} n_{*} \Delta \phi_{\mathrm{pm}}} .
$$

Random stars begin to cross the uncertainty ellipse at $\bar{\theta} \sim \pi$, which sets the limiting distance $r \sim L_{3 \mathrm{a}}$ at

$$
L_{3 \mathrm{a}} \sim\left(\frac{3}{2 \pi n_{*} \Delta \phi}\right)^{1 / 3} \text {. }
$$

Then, with $\Delta \phi_{\mathrm{pm}} \sim \epsilon_{\mathrm{pm}} L_{3 \mathrm{a}} / u_{*}$,

$$
L_{3 \mathrm{a}} \sim\left(\frac{3 u_{*}}{2 \pi n_{*} \epsilon_{\mathrm{pm}}}\right)^{1 / 4} .
$$

Using solar neighborhood parameters for $u_{*}$ and $n_{*}$, with $\epsilon_{\mathrm{pm}} \sim 1$ mas $\mathrm{yr}^{-1}$ for the TGAS Hipparcos subset, gives $L_{3 \mathrm{a}} \sim 10 \mathrm{pc}$, and a corresponding $\tau_{3 \mathrm{a}} \sim 0.5 \mathrm{Myr}$.

The second criterion has the effect of reducing $n_{*}$ by factor $\kappa_{3 \mathrm{~b}}$ set by the uncertainty $\epsilon_{\perp}$ in $u_{\perp} \equiv r \tilde{u}$ relative to the spread $\sigma_{v}$ 
in $v_{\infty} \sin \theta_{0 *}$. Then,

$$
\begin{aligned}
\epsilon_{\perp} & =u_{\perp}\left(\left(\frac{\epsilon_{\mathrm{pm}}}{\tilde{u}}\right)^{2}+\left(\frac{r \epsilon_{\mathrm{plx}}}{\alpha}\right)^{2}\right)^{1 / 2} \\
& \sim \frac{r u_{*}}{\alpha}\left(\hat{\epsilon}_{\mathrm{pm}}^{2}+\epsilon_{\mathrm{plx}}^{2}\right)^{1 / 2} .
\end{aligned}
$$

Next, with $\sigma_{v} \sim v_{\infty} \sim u_{*}$,

$$
\kappa_{3 \mathrm{~b}} \sim \frac{\epsilon_{\perp}}{\sigma_{v}} \sim \frac{r}{\alpha}\left(\hat{\epsilon}_{\mathrm{pm}}^{2}+\epsilon_{\mathrm{plx}}^{2}\right)^{1 / 2} .
$$

Finally, substitute $r \rightarrow L_{3}, n_{*} \rightarrow \kappa_{3 \mathrm{~b}} n_{*}$, and $L_{3 \mathrm{a}} \rightarrow L_{3}$ in Equation (17) to find

$$
L_{3} \sim\left(\frac{3 \alpha^{2}}{2 \pi n_{*} \hat{\epsilon}_{\mathrm{pm}}}\right)^{1 / 5}\left(\hat{\epsilon}_{\mathrm{pm}}^{2}+\epsilon_{\mathrm{plx}}^{2}\right)^{-1 / 10},
$$

which gives $L_{3} \sim 30 \mathrm{pc}$ and $\tau_{3} \sim 1.5 \mathrm{Myr}$. Since $L_{3} \sim L_{2}^{\prime}$ and $\tau_{3} \sim \tau_{2}^{\prime}$, this method is comparably capable at identifying encounters as a method using six-parameter solutions for each star with well-constrained radial velocities. Thus, as long as uncertainties in stellar astrometry permit the use of the linear motion approximation, these geometric criteria serve as an effective means to identify any past encounter of high statistical confidence with minimal computational power.

\subsection{Search Results}

A search of TGAS for a potential origin of 1I was designed and conducted with consideration of the uncertainties and limits discussed above. Stars were filtered by the geometric criteria specified above, with a successful match is defined by a $3 \sigma$ overlap in uncertainties. The uncertainty ellipse for the origin direction of 1I is approximated as completely flattened with minor axis $\Delta \phi_{2}=0$ to simplify computation. Only stars with nominal distance $r_{0}<r_{\max }=10 \mathrm{pc}$ from the Sun-well within the computed $L_{3}$-were considered. None of the 68 stars considered matched these criteria, indicating that the parent star of 1I cannot be conclusively identified with TGAS.

This null result does not necessarily imply that 1I originated from beyond this region. While TGAS contains most stars in the immediate solar neighborhood of the characteristic $\mu_{*} \sim 0.2 G M_{\odot}$ and above, it does not constitute a comprehensive catalog of planetary systems in this region. Even brown dwarfs_completely absent in TGAS-have been observed with protoplanetary disks from which ISOs could plausibly originate (Apai et al. 2005), and moreover, may be as abundant as stars (Chabrier 2003). The full Gaia DR1 samples these objects, with full astrometric solutions expected in upcoming data releases, albeit with limited completeness beyond a few parsecs (de Bruijn 2014).

Note that including these low-mass stars and substellar brown dwarfs raises $n_{*}$ - perhaps doubling its value-but does not appreciably change the estimated $L_{1}, L_{2}^{\prime}$, and $L_{3}$ (and their corresponding timescales), which vary extremely slowly with $n_{*}$. These objects should also contribute only minimally to ISO scattering and $L_{0}$, which, as discussed earlier, is primarily constrained by stars of $\mu_{*} \sim 0.2 G M_{\odot}$.

Finally, as a consistency check of the estimated limiting distance $L_{3}$, the search was repeated with all stars in TGAS (i.e., $\left.r_{\max } \rightarrow \infty\right)$. The nearest star matching both geometric criteriapresumably by random chance-had a nominal $r_{0} \approx 40.5 \mathrm{pc}$. This distance is fairly close to the $r_{0} \sim L_{3} \sim 30 \mathrm{pc}$ expected of

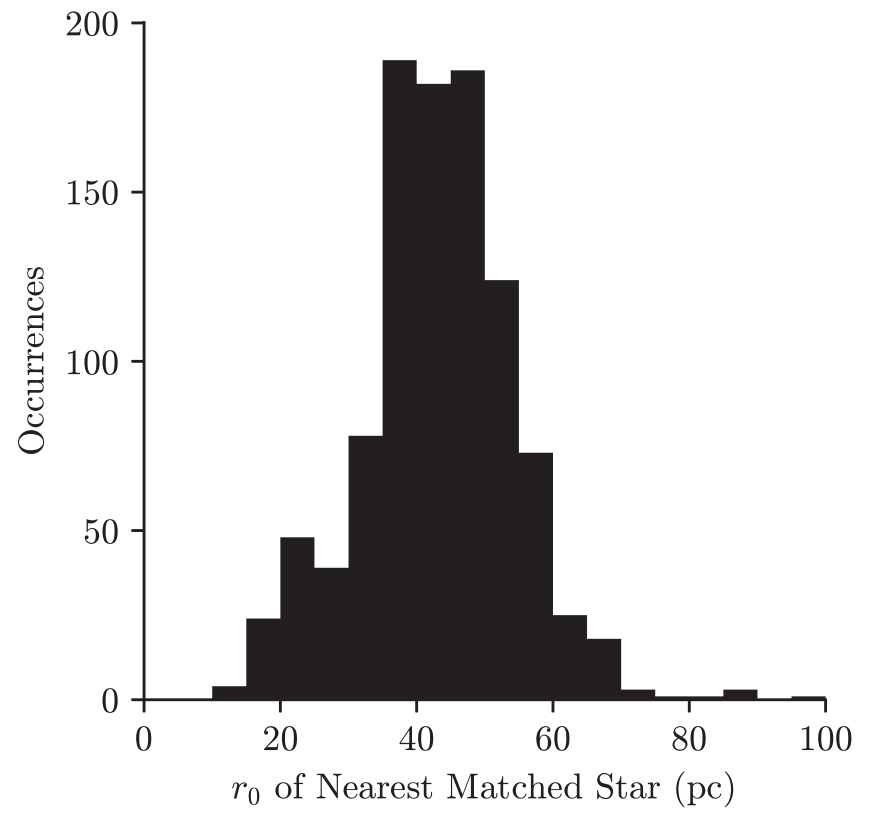

Figure 3. Distribution of nominal distance $r_{0}$ for the nearest star matched by the introduced geometric search criteria (bin size: 5 pc) over a sample of 1000 pseudo-randomly generated ISOs with approach velocities distributed according to the local stellar velocity dispersion, but with relative uncertainties $\left(\Delta \phi_{1}\right.$, $\Delta \phi_{2}$, and $\Delta \phi_{3}$ ) matching those of 1I's trajectory. This distribution is consistent with the predicted $r_{0} \sim L_{3} \sim 30$ pc for the nearest match in a typical search.

the nearest random matching star for an arbitrary ISO with trajectory uncertainties $\Delta \phi_{1}, \Delta \phi_{2}$, and $\Delta \phi_{3}$ identical to those of $1 \mathrm{I}$.

To ensure that this result is not unique to 1I's trajectory, this procedure was repeated, replacing 1I with a sample of 1000 ISOs with pseudo-randomly generated nominal approach trajectories. These trajectories were produced by selecting its galactic $U, V$, and $W$ components of velocity from normal distributions matching those of the local stellar velocity dispersion (Huang et al. 2015). Results are plotted in Figure 3, which shows that the distribution of $r_{0}$ for the nearest star matching the criteria is approximately normal, with mean $42.9 \pm 0.4 \mathrm{pc}$ and standard deviation $11.2 \pm 0.3 \mathrm{pc}$. These results are, again, roughly consistent with the analytically estimated $r_{0} \sim L_{3} \sim 30 \mathrm{pc}$ for the nearest match of a typical search, to within a factor of two.

\section{Conclusions}

1I is the first, but likely not the last ISO to be discovered in the solar system. Forthcoming surveys like LSST will likely provide additional opportunities to study these objects and, in effect, the distant environments in which they form. The capability to match an ISO to its original planetary system would provide a unique mode to examine specific extrasolar planetary systems at close proximity.

The preceding sections showed that trajectory analysis alone is unlikely to be successful for any particular ISO, although the possibility of occasional success cannot be excluded. Gravitational scattering from random stellar encounters limits traceability to the past few $10 \mathrm{Myr}$. Observational bias favoring young ISOs may elevate the fraction of discovered ISOs that are potentially traceable above the $<1 \%$ of galactic history contained by this limit. More stringent limits, however, are placed by astrometric uncertainties associated with both the 
ISO and the stars. These uncertainties become important when velocity uncertainties greatly exceed one part in $10^{5}$-an angular uncertainty of a few arcseconds-including in the present case of $1 \mathrm{I}$.

Currently, stellar astrometry provides the limiting constraint, with TGAS enabling the positive identification of a close encounter out to just a few parsecs, spanning only the immediate solar neighborhood. A search conducted for 1I of TGAS stars presently within this region fails to produce such a positive identification, indicating that the ISO must have originated from either a nearby low-mass star or brown dwarf not in TGAS, or from a more distant star outside of the region that cannot, at present, be identified. This result does not preclude searches for candidates beyond the limited regionincluding those found by Gaidos et al. (2017), Portegies Zwart et al. (2017), Dybczyński \& Królikowska (2017), and Feng \& Jones (2017) _ but merely implies that such candidates are unlikely to be linkable to the ISO's trajectory with reasonable statistical confidence. Improvements to stellar astrometric uncertainty are anticipated with the upcoming Gaia data releases, which are expected to significantly expand this range and improve catalog completeness in the near future.

The author thanks Quan-Zhi Ye, Shreyas Vissapragada, Yayaati Chachan, and Konstantin Batygin for insightful discussions on the potential origins of 1I. Special thanks to an anonymous referee whose comments and suggestions helped improve this manuscript. This research has made use of data and/ or services provided by the International Astronomical Union's Minor Planet Center, and by the Jet Propulsion Laboratory's Solar System Dynamics Group. This work has made use of data from the European Space Agency (ESA) mission Gaia (https:// www.cosmos.esa.int/gaia), processed by the Gaia Data Processing and Analysis Consortium (DPAC, https://www.cosmos. esa.int/web/gaia/dpac/consortium). Funding for the DPAC has been provided by national institutions, in particular, the institutions participating in the Gaia Multilateral Agreement.
Software: Matplotlib (Hunter 2007), NumPy (Walt et al. 2011), Python (van Rossum 1995).

\section{ORCID iDs}

Qicheng Zhang (1D https://orcid.org/0000-0002-6702-191X

\section{References}

Apai, D., Pascucci, I., Bouwman, J., et al. 2005, Sci, 310, 834 Chabrier, G. 2003, PASP, 115, 763

de Bruijn, J. 2014, MmSAI, 85, 631

Dybczyński, P. A., \& Królikowska, M. 2017, A\&A, submitted (arXiv:1711. 06618)

Engelhardt, T., Jedicke, R., Vereš, P., et al. 2017, AJ, 153, 133

Feng, F., \& Jones, H. R. A. 2017, ApJL, in press (arXiv:1711.08800)

Gaidos, E., Williams, J., \& Kraus, A. 2017, RNAAS, 1, 13

Gaia Collaboration, Brown, A. G., Vallenari, A., et al. 2016b, A\&A, 595, A2 Gaia Collaboration, Prusti, T., de Bruijne, J. H. J., et al. 2016a, A\&A, 595, A1 Huang, Y., Liu, X.-W., Yuan, H.-B., et al. 2015, MNRAS, 449, 162 Hunter, J. D. 2007, CSE, 9, 90

Innanen, K., Patrick, A., \& Duley, W. 1978, Ap\&SS, 57, 511

Jewitt, D., Luu, J., Rajagopal, J., et al. 2017, ApJL, 850, L36

Kaiser, N., Aussela, H., Burkeb, B., et al. 2002, Proc. SPIE, 4836, 155

Kennedy, G. M., \& Kenyon, S. J. 2008, ApJ, 673, 502

Kennicutt, R. C., Jr, \& Evans, N. J. 2012, ARA\&A, 50, 531

Mamajek, E. 2017, RNAAS, 1, 21

Meech, K. J., Weryk, R., Micheli, M., et al. 2017, Natur, 552, 378

Michalik, D., Lindegren, L., \& Hobbs, D. 2015, A\&A, 574, A115

Portegies Zwart, S., Pelupessy, I., Bedorf, J., Cai, M., \& Torres, S. 2017, MNRAS, submitted, (arXiv:1711.03558)

Sekanina, Z. 1976, Icarus, 27, 123

Sen, A., \& Rana, N. 1993, A\&A, 275, 298

Trilling, D. E., Robinson, T., Roegge, A., et al. 2017, ApJL, 850, L38 Tyson, J. A. 2002, Proc. SPIE, 4836, 10

van Rossum, G. 1995, Python Reference Manual, Report CS-R9525 (Amsterdam: Centrum voor Wiskunde en Informatica)

Walt, S. V. D., Colbert, S. C., \& Varoquaux, G. 2011, CSE, 13, 22

Williams, G. V. 2017a, MPEC, 2017-U183

Williams, G. V. 2017b, MPEC, 2017-U181

Williams, G. V. 2017c, MPEC, 2017-V17

Ye, Q.-Z., Zhang, Q., Kelly, M. S. P., \& Brown, P. G. 2017, ApJL, 851, L5 